\title{
New Results from the Modeling of the Shell around IRC +10216
}

\author{
M. A. T. GROENEWEGEN \\ Max-Planck Institut für Astrophysik, Garching, Germany
}

\begin{abstract}
A spherically symmetric dust radiative transfer code is used to model the circumstellar dust shell around IRC +10216 . Compared to numerous previous models a much larger body of observational data is used as constraints. The spectral energy distribution between 0.5 and $60000 \mu \mathrm{m}, 2-4 \mu \mathrm{m}$ and 8-23 $\mu \mathrm{m}$ spectra, optical, far-infrared and centimeter sizes, and interferometric visibility curves between 1.6 and $11.2 \mu \mathrm{m}$ are used to constrain the model.
\end{abstract}

Key results are:

- In order to fit the visibility curve at $2.2 \mu \mathrm{m}$ and the size of the shell in the optical, scattering has to be invoked. The strong dependence of the scattering coefficient on grain size allows one to derive a mean grain size of $0.16 \pm 0.01 \mu \mathrm{m}$.

- Previous suggestions that the mass loss rate was higher in the past are confirmed. The principal argument is that with an $r^{-2}$ model the calculated far-infrared sizes are smaller than observed.

- Regarding the cm emission it is found that in small apertures dust emission is negligible for wavelengths $\gtrsim 2 \mathrm{~cm}$. Free-free emission is negligible for wavelengths $\lesssim 0.5 \mathrm{~cm}$. The free-free emission is found to be optically thin even at $6 \mathrm{~cm}$. An ionization fraction of $7.8 \times 10^{-5}$ is derived which, according to the Saha equation, corresponds to an electron temperature of about $2400 \mathrm{~K}$. Although there are uncertainties in the free-free emission model, this suggests that the free-free emission does not come from a chromosphere.

This research is discussed further in Groenewegen (1996, $A \mathcal{E} A, 305, \mathrm{~L} 61)$ and Groenewegen (1997, $A \& A, 317,503)$. 